\title{
Traumatic Optic Nerve Sheath Hematoma
}

\author{
Aria Ghahramani $^{a}$ Mona L. Camaccib Rucha Borkhetaria ${ }^{a}$ \\ Anne Poulsen $^{\mathrm{b}}$ Samuel Beckstead ${ }^{\mathrm{b}}$ Christopher Weller ${ }^{\mathrm{b}}$ \\ aPenn State College of Medicine, Hershey, PA, USA; bepartment of Ophthalmology, Penn \\ State College of Medicine, Hershey, PA, USA
}

\section{Keywords}

Optic nerve $\cdot$ Trauma $\cdot$ Traumatic optic neuropathy

\begin{abstract}
The aim of this report is to present a patient with traumatic optic nerve sheath hematoma $(\mathrm{ONSH})$, a rare diagnosis with high potential for visual sequelae. This case involves a 41-yearold male who presented promptly following blunt trauma to the right eye and orbit that resulted in acute vision loss. Following computed tomography and ophthalmic examination, a diagnosis of ONSH was made and medical therapy with methylprednisolone was initiated. He reported significant improvements in visual symptoms following intravenous corticosteroid therapy. Although the patient reported significant improvements and had normal Snellen visual acuities in follow-up, he continued to have an inferior visual field defect at 1 week in the affected eye. ONSH causing subsequent localized compression of the optic nerve is a rare mechanism of traumatic optic neuropathy in patients following head trauma. The localized compartment syndrome of the optic nerve and subjective visual symptoms were relieved following corticosteroid therapy with no initial need for surgical decompression. Although central visual acuity returned to baseline, the patient had a persistent visual field defect and relative afferent pupillary defect.
\end{abstract}

\section{Introduction}

Although rare, traumatic optic neuropathy (TON) is a reported sequela of significant facial trauma. Previous epidemiological studies have shown that TON presents in roughly $0.4 \%$ of all trauma patients, particularly in patients who experience head injuries [1]. TON results from direct or indirect forces causing injury to the optic nerve. Causes of direct TON 
include transection, avulsion, orbital hemorrhage, orbital emphysema, and optic nerve sheath hemorrhage [2, 3].

TON is associated with decreased visual acuity (VA), with $40-60 \%$ of patients presenting with light perception or worse $[4,5]$. Patients with TON often present with relative afferent pupillary defect (RAPD), impaired color vision, and variable visual field defects [6]. Patients with anterior optic nerve injury could have abnormalities such as swelling of the optic nerve head with adjacent retinal hemorrhages, and however, posterior optic nerve injury may go unnoticed on routine fundus exam [6].

Optic nerve sheath hematoma (ONSH) causes direct compression on the optic nerve. It is an unusual cause of TON in patients presenting with acute vision loss following a trauma. In addition to direct compression, these hematomas may produce a localized compartment syndrome from hemorrhage contained within the barrier formed by the optic nerve sheath [7]. Computed tomography (CT) is the preferred radiological method for the evaluation of ocular and orbital trauma and allows visualization of the hematoma surrounding the optic nerve sheath $[8,9]$.

Treatment for TON in general aims to relieve the localized compartment syndrome or source of direct compression and improve tissue edema and inflammation. Interventions previously reported range from conservative management, high-dose intravenous steroids, decompressive surgery, or a combination of steroids and surgery $[10,11]$. In the setting of acute TON, better outcomes are reported in cases of early intervention as well as those with edema or hematoma of the optic nerve sheath, as compared to those patients with fracture lines through the optic canal or delayed interventional time longer than 24-h after trauma [4]. Based off their experience, $\mathrm{Wu}$ and colleagues [11] recommend methylprednisolone $250 \mathrm{mg}$ delivered intravenously, ideally, within $8 \mathrm{~h}$ after trauma. It is then further dosed every $6 \mathrm{~h}$ for a total of $48 \mathrm{~h}$. Their team also recommended optic canal decompression within 12-24 h of beginning of medical therapy [11]. Conversely to the previously reported improved outcomes with early intervention, recent systematic reviews of high-dose corticosteroids and optic canal decompression have shown that neither treatment provides convincing visual benefit over observation alone and could be harmful in patients with concomitant head trauma [12, 13]. Given the rarity of ONSHs, no significant large-scale studies have compared the various treatment options and their effects on visual outcome [2]. While there is no single optimal approach to TON, there may be specific exam findings, such as the presence of an ONSH that might benefit from particular treatments such as high-dose corticosteroids.

\section{Case Report}

A 41-year-old male presented to a Level 1 Emergency Department as a transfer after a traumatic injury to the right eye. The patient reported that he was repairing farming equipment when a pair of pliers slipped and hit him in the right eye. He reported experiencing complete vision loss in the right eye for several minutes. At time of ophthalmic examination, $8 \mathrm{~h}$ from time of injury, patient reported that although his vision had slowly returned and was back to normal in the upper portion of his visual field, the lower portion remained "darker and hazy."

External examination was significant for pronounced periorbital ecchymosis and edema on the right. Ocular examination revealed a VA of 20/40 on the right and 20/25 on the left on Snellen chart. 10/11 plates were identified in the right eye and 11/11 in the left via Ishihara subjective color testing. Confrontational visual field revealed depression in the inferior quadrants in the right eye. Intraocular pressure was $16 \mathrm{~mm} \mathrm{Hg}$ in the right eye and $11 \mathrm{~mm} \mathrm{Hg}$ in the left. Pupil examination was significant for a $2+$ rAPD in the right eye. Motility examination in the right eye revealed $30 \%$ restriction of ocular movements in all fields of gaze. Slit lamp and dilated 
fundus examination of both eyes revealed scattered subconjunctival hemorrhage of the right eye but otherwise was unremarkable with no retinal or disc edema on fundoscopic exam. The patient did not have macular or retinal nerve fiber OCT or fundus photography at presentation.

An initial noncontrast CT scan at an outside hospital showed retrobulbar hemorrhage with mild proptosis (shown in Fig. 1). A repeat CT scan performed $7 \mathrm{~h}$ later revealed a stable hematoma surrounding the optic nerve within the optic nerve sheath with surrounding fat stranding and a superior orbital rim fracture with a fragment displaced into the orbit. Based on clinical and imaging findings, a diagnosis of ONSH secondary to blunt facial trauma was made. Given the minimally decreased VA of the affected eye, stability of serial examinations, location of the hematoma, and risks of potential surgical drainage, the decision was made to defer acute surgical intervention in favor of medical therapy with intravenous methylprednisolone $250 \mathrm{mg}$ q6h for $24 \mathrm{~h}$ with continued close observation. Canthotomy/cantholysis was initially considered as a potential treatment option, but given the normal ocular pressure and no tightness of the lids, decision was made that a Canthotomy/cantholysis would not release any pressure.

Clinical improvement was observed within $24 \mathrm{~h}$. Periorbital edema subsided, and VA in the right eye returned to baseline at 20/20 with a 2+ rAPD and subjective residual inferior confrontational field deficit. Ishihara subjective color test was normal 11/11 in both eyes. The patient was discharged with oral prednisolone $10 \mathrm{mg}$ for 2 days, and oral acetaminophen 325 mg PRN for pain. At 1-week follow-up, the patient reported no difference between superior and inferior visual fields. VA in the right eye was 20/20, and 20/25 in the left. The patient had a small improvement on pupillary exam with a 1+rAPD in the right eye. Humphrey visual field 30-2 revealed a lower visual field defect on the right (shown in Fig. 2). Patient was scheduled for a 3-month follow-up with plans for repeat Humphrey visual field, and however, no subsequent visual field was obtained as the patient was lost to follow-up.

\section{Discussion}

This report describes an unusual presentation of TON in which a compressive ONSH resulted from blunt facial trauma with improvement of visual symptoms following IV corticosteroid therapy. The rarity of TON and the compartment syndrome that may follow requires prompt treatment following injury. The decision to administer conservative therapy, intravenous steroids, decompressive surgery, or a combination of steroids and surgery remains based on a case-by-case approach [14]. Wu and colleagues [11] have advised treatment with methylprednisolone if a patient presents within $8 \mathrm{~h}$ of insult and optic canal decompression

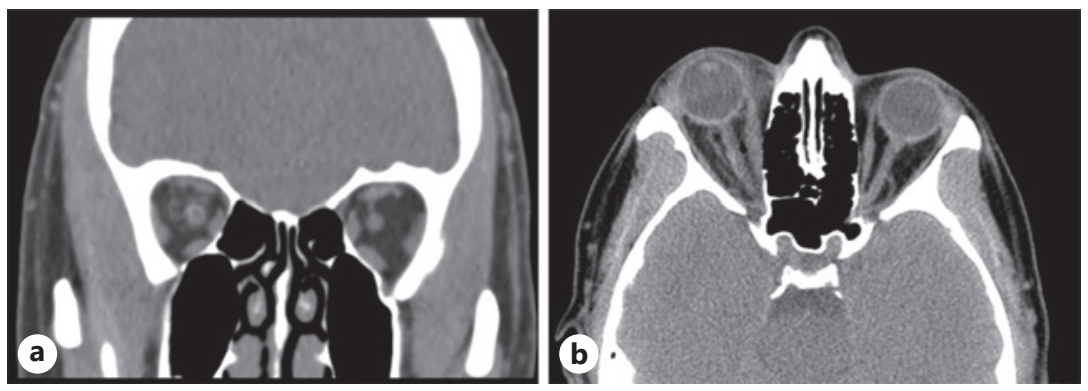

Fig. 1. a Coronal noncontrast CT orbit demonstrating a right orbital nerve sheath hemorrhage and adjacent fat stranding. Arrow demonstrating the ONSH. b Axial noncontrast CT orbits demonstrating a right orbital nerve sheath hemorrhage and adjacent fat stranding. Proptosis of right eye visible. Arrow demonstrating the ONSH. ONSH, Optic nerve sheath hematoma; CT, computed tomography. 


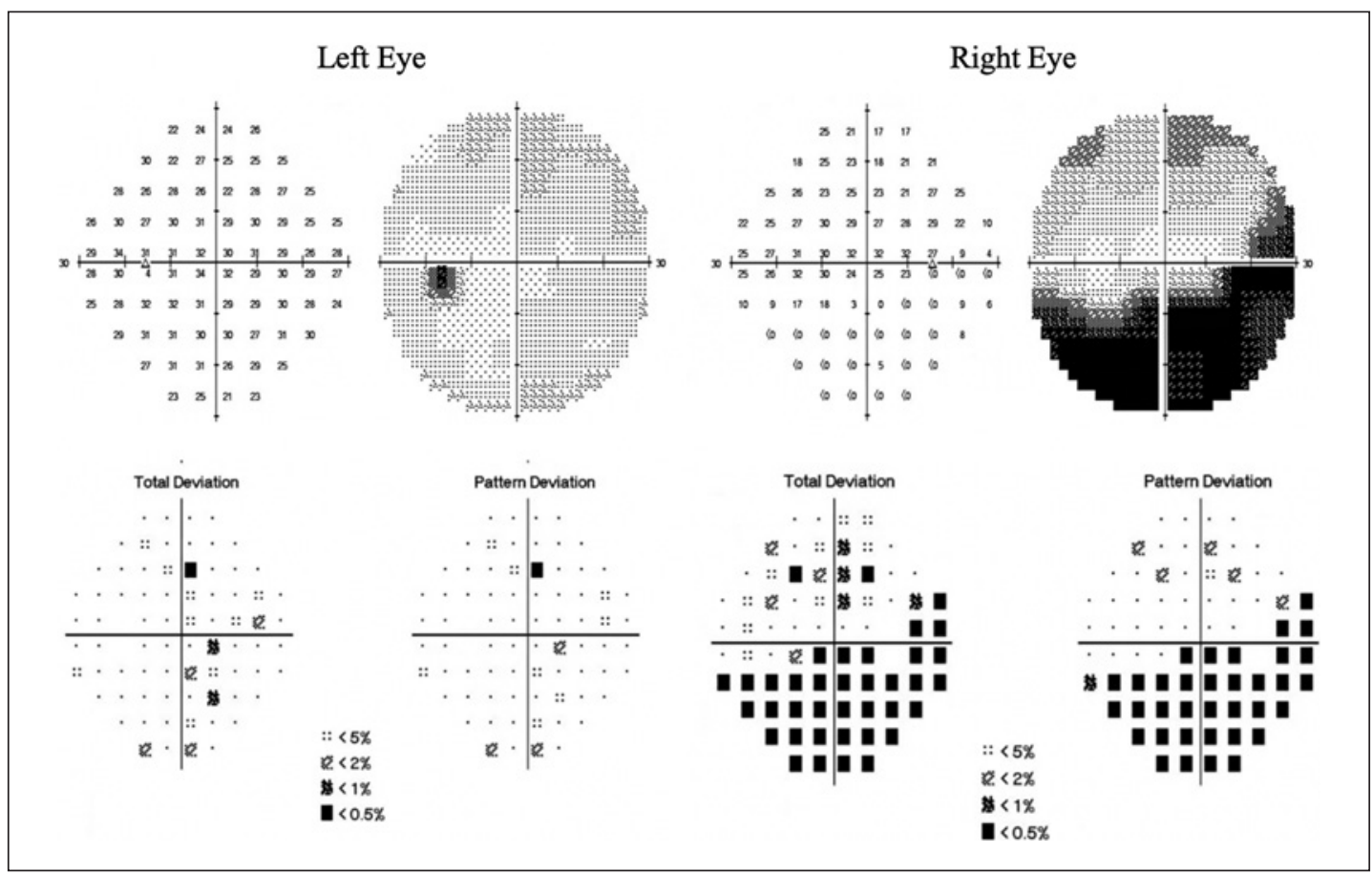

Fig. 2. HVF 30-2 at 1-week post-injury revealing a lower visual field defect in the right eye. HVF, Humphrey visual field.

within 12-24 h of beginning medical treatment. Here, we report full central vision recovery within $24 \mathrm{~h}$ of starting IV administration of methylprednisolone in a patient who was treated within $8 \mathrm{~h}$ of initial insult, and whose CT showed hematoma surrounding the optic nerve sheath. As the cause of the optic neuropathy was presumed to be secondary to the nerve sheath hematoma within the orbit, there was no indication for optic canal decompression surgery. However, patient did continue to have an inferior field defect at follow-up and was subsequently lost to further follow-up.

This report describes an unusual case of TON in which a compressive ONSH caused a potential localized compartment syndrome from hemorrhage within the sheath. Treatment with high-dose IV corticosteroids resulted in rapid and sustained recovery of visual symptoms. These findings suggest that medical management of TON with ONSH could be effective when patients present promptly following trauma and are closely monitored with no signs of decompensation. It is worth noting that relatively high rates of spontaneous visual recovery following TON have been reported in the literature [15]. The resolution of the ONSH and improvement in VA may have been a natural progression with spontaneous evolution rather than due to the implementation of the steroid therapy. The present case report does not prove the efficacy of steroid therapy for management of TON but provides a case in which symptoms resolved, adverse outcomes were avoided, and surgical intervention was not needed following administration of steroids.

\section{Statement of Ethics}

Consent to publish was not obtained. This report does not contain any personal information that could lead to the identification of the patient. The authors of this report obtained a waiver from the Institutional Review Board (IRB). Per the IRB, this study does not meet the 
definition of research as per the Office for Human Research Protections 45 CFR 46.102(l), and as such, does not require review by an IRB. The team confirmed that after several attempts they were unable to reach the patient on whom the case report is written, and the manuscript as drafted does not include any identifiable information. This is in compliance with institutional guidelines, as outlined on page 38 of HRP-103 - Investigator Manual and no further action is necessary at this time.

\section{Conflict of Interest Statement}

The authors have no conflicts of interest to declare.

\section{Funding Sources}

No funding or grant support was received for this research.

\section{Author Contributions}

M.C. acquired the patient data and interpreted it. A.G., M.C., and R.B. drafted the manuscript. M.C., A.P., S.B., and C.W. revised the manuscript critically for important intellectual content. All the authors read and approved the final version of the manuscript to be published.

\section{References}

1 Pirouzmand F. Epidemiological trends of traumatic optic nerve injuries in the largest Canadian adult trauma center. J Craniofac Surg. 2012;23(2):516-20.

2 Warner N, Eggenberger E. Traumatic optic neuropathy: a review of the current literature. Curr Opin Ophthalmol. 2010;21(6):459-62.

3 Sarkies N. Traumatic optic neuropathy. Eye. 2004;18(11):1122-5.

4 Urolagin SB, Kotrashetti SM, Kale TP, Balihallimath LJ. Traumatic optic neuropathy after maxillofacial trauma: a review of 8 cases. J Oral Maxillofac Surg. 2012;70(5):1123-30.

5 Spoor TC, Hartel WC, Lensink DB, Wilkinson MJ. Treatment of traumatic optic neuropathy with corticosteroids. Am J Ophthalmol. 1990;110(6):665-9.

6 Kumaran AM, Sundar G, Chye LT. Traumatic optic neuropathy: a review. Craniomaxillofac Trauma Reconstr. 2015;8(1):31-41.

7 Steinsapir KD, Goldberg RA. Traumatic optic neuropathy. Surv Ophthalmol. 1994;38(6):487-518.

8 Mine S, Yamakami I, Yamaura A, Hanawa K, Ikejiri M, Mizota A, et al. Outcome of traumatic optic neuropathy: comparison between surgical and nonsurgical treatment. Acta Neurochir. 1999;141(1):27-30.

9 Lee HJ, Jilani M, Frohman L, Baker S. CT of orbital trauma. Emerg Radiol. 2004;10(4):168-72.

10 Levin LA, Beck RW, Joseph MP, Seiff S, Kraker R. The treatment of traumatic optic neuropathy: the international optic nerve trauma study. Ophthalmology. 1999;106(7):1268-77.

11 Wu N, Yin ZQ, Wang Y. Traumatic optic neuropathy therapy: an update of clinical experimental studies. J Int Med Res. 2008;46(5):883-9.

12 Yu-Wai-Man P, Griffiths PG. Steroids for traumatic optic neuropathy. Cochrane Database Syst Rev. 2013 Jun; (6):CD006032.

13 Yu-Wai-Man P, Griffiths PG. Surgery for traumatic optic neuropathy. Cochrane Database Syst Rev. 2005 Oct; 6(4):CD005024.

14 Kaštelan S, Gverović AA, Salopek RJ, Gotovac M, Orešković D, Kasun B. Traumatic optic neuropathy: case report with discussion on diagnostic procedures and therapy. Acta Clin Croat. 2018;57(1):166-72.

15 Yu-Wai-Man P. Traumatic optic neuropathy-clinical features and management issues. Taiwan J Ophthalmol. 2015;5(1):3-8. 